\title{
A Quantum Tango between Magnons and Phonons
}

\author{
A nanopatterned magnetic structure features an unprecedently strong \\ coupling between lattice vibrations and quantized spin waves, which \\ could lead to novel ways of manipulating quantum information.
}

\author{
By Cassidy R. Berk and Holger Schmidt
}

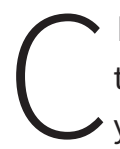

hanges in information storage technologies reflect the increasing complexity of societies [1]. Five thousand years ago, the progress of agricultural civilizations in Mesopotamia drove the development of the cuneiform script-wedge-shaped carvings on clay tablets. Today, innovative strides of the information society have led to spintronic devices that "carve" 0's and 1's into the spin states of

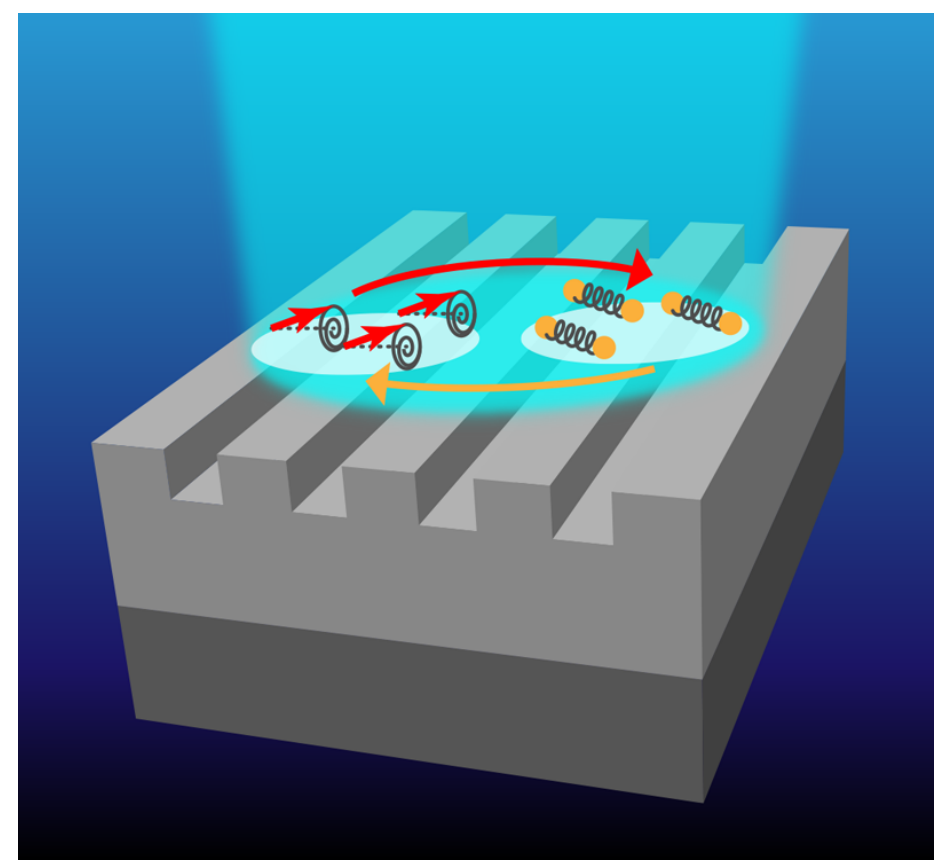

Figure 1: In a nanopatterned magnetic structure illuminated by a short laser pulse, magnons and photons couple to form quasiparticles called magnon-polarons.

Credit: APS/Alan Stonebraker electrons in magnetic materials. But our growing hunger for data calls for even denser and faster storage methods. Magnonics is a promising next-generation approach in which quantized spin waves, or magnons, are used to carry information. Magnons are generally hard to manipulate, but a study by Felix Godejohann of the Technical University of Dortmund, Germany, and colleagues could lead to new ways to control magnons via their coupling to acoustic vibrations, or phonons, in a material [2]. The researchers engineered a grooved magnetic structure that allowed them to study and optimize various factors affecting magnon-phonon coupling, achieving an unprecedented coupling strength.

The use of magnons as information carriers entails several prospective benefits. Their high frequencies ( $\mathrm{GHz}$ to $\mathrm{THz}$ ) and short wavelengths could lead to faster and smaller memory elements, compared with electron-based circuits [3]. What's more, magnonics could be free from some of the inherent drawbacks of electronics because it doesn't require propagating electron currents that are inevitably associated with heating and energy loss.

Even more intriguing are the prospects of using magnons in hybrid quantum information schemes, in which coherent quantum states are shuttled between different systems-superconducting qubits, atoms, ions, and electron spins [4]. This coherent transduction requires ways to efficiently couple various elementary excitations to one another. Magnons can conveniently couple to microwave photons, which can interact, for instance, with atoms and ions. Phonons could perform a similar role, as they could mediate the transduction from magnons to electrons [5]. 
Both classical and quantum information applications have thus motivated intense research efforts aimed at understanding and controlling magnon-phonon interaction [5]. Promising platforms for achieving the desired interaction strength are offered by magnetostrictive materials, in which magnetization and mechanical strain are associated. In these materials, spin-orbit coupling associates lattice deformations (phonons) with changes to the spin system (magnons). Much of recent research has focused on exciting magnons through phonons-a one-way transfer of energy $[6,7]$ (see Focus: Sound-Driven Spin Waves). But if the mutual interaction is sufficiently strong, magnons and phonons can partake in a more intimate dance with one another, with a back-and-forth energy transfer between them. This "tango" results in the formation of a magnon-polaron, a hybrid quasiparticle that is neither magnon nor phonon.

The formation of this quasiparticle has a characteristic experimental signature. In the absence of coupling, the magnons and phonons would have the same frequency-their energies are degenerate. When coupling is sufficiently strong, the mutual interaction between the magnons and phonons lifts the degeneracy and leads to the formation of two energy bands that are split. This splitting depends on the strength of hybridization, which is quantified by $C$, the cooperativity parameter. The value of $C$ depends on how fast the phonons and magnons exchange energy, compared to how fast they independently lose energy. If $C>1$, the system is in the strong-coupling regime.

Until recently, engineering a device able to operate in this strong-coupling regime remained an elusive goal. But in 2019, our group reported the first unequivocal observation of a strongly coupled magnon-polaron quasiparticle in a nanomagnet. The hybridization was obtained by tuning the magnet's magnon mode with an external field so that its frequency matched one of the vibrational resonances of the nanomagnet. Under optimal orientation of the applied field with respect to the magnet, we reached a strong-coupling regime with $C$ as high as 1.7 [8].

Godejohann and colleagues have brought the coupling strength to new heights and have shed light on the factors that contribute to it. The team devised a clever scheme by which they could tweak the various factors contributing to magnon-phonon hybridization. They used a thin film of Galfenol-a highly magnetostrictive alloy of iron and gallium-and carved 7-nm-thick parallel grooves onto the film's surface, producing a grating-like pattern with a $200-\mathrm{nm}$ period (Fig. 1). Such a grating affects the spatial distribution of both magnons and phonons, whose overlap influences the strength of their mutual coupling.

The team used a pump-probe experiment to observe in real time the effects of the magnon-phonon hybridization. A short "pump" laser pulse hit the sample, exciting both magnons and a set of phonons with different frequencies. A second "probe" pulse then measured the sample's reflectivity, whose dynamics carried the signatures of the phonon and magnon oscillations in the film. With an applied magnetic field, the researchers could tune the frequency of a specific magnon mode, which allowed them to observe the sample response as this frequency was scanned through the vibrational resonances. They found that tuning the magnon mode to a specific phonon mode led to a clear band splitting that indicated strong hybridization ( $C$ of about 8). Tuning the magnon frequency to that of another phonon mode, however, enhanced the strength of the magnon resonance but didn't lead to observable hybridization.

Through data analysis and numerical simulations, the team derived key conclusions on what boosts magnon-phonon coupling. First, the hybridization of the modes is strongest when the modes' spatial distributions match. Second, a balanced strength of the modes is crucial to creating a hybridized state: When the excitation is concentrated on the phonons, they dominate the dynamics of the system and hybridization is not observed.

The elegant scheme is well suited to explore properties of magnon-polarons from both a fundamental and an applied perspective. Experiments with similar configurations could explore the transport and scattering properties of these quasiparticles in view of their potential use as quantum transducers. Furthermore, since different types of magnon-polarons can be distinguished by their spatial localization or their frequency, one can envision multiplexing schemes that encode a complex signal into multiple quasiparticles. Another interesting direction would be to involve microwave photons in the dance. Researchers have demonstrated coupling involving photons, phonons, and 
magnons in 3D cavities, and used it to realize effects such as electromagnetically induced transparency, parametric amplification, and phonon lasing [9]. Compared to cavity-based schemes, the planar geometry of Godejohann and colleagues' structure is better suited for applications in integrated optoelectronic circuits.

Cassidy R. Berk: Lawrence Berkeley National Laboratory, Berkley, California, USA

Holger Schmidt: Baskin School of Engineering, University of California, Santa Cruz, USA

\section{REFERENCES}

1. Y. N. Harari, Sapiens: A Brief History of Humankind (Harper, New York, 2015).

2. F. Godejohann et al., "Magnon polaron formed by selectively coupled coherent magnon and phonon modes of a surface patterned ferromagnet," Phys. Rev. B 102, 144438 (2020).

3. A. V. Chumak et al., "Magnon spintronics," Nat. Phys. 11, 453 (2015).

4. G. Li et al., "Li, An, and Morozov Reply:," Phys. Rev. Lett. 123, 119602 (2019).

5. D. A. Bozhko et al., "Magnon-phonon interactions in magnon spintronics," Low Temp. Phys. 46, 383 (2020).

6. P. G. Gowtham et al., "Mechanical back-action of a spin-wave resonance in a magnetoelastic thin film on a surface acoustic wave," Phys. Rev. B 94, 014436 (2016).

7. W. G. Yang et al., "Magnetoelastic excitation of single nanomagnets for optical measurement of intrinsic Gilbert damping," Phys. Rev. B 97, 224410 (2018).

8. C. Berk et al., "Strongly coupled magnon-phonon dynamics in a single nanomagnet," Nat. Commun. 10, 2652 (2019).

9. X. Zhang et al., "Cavity magnomechanics," Sci. Adv. 2, e1501286 (2016). 